\title{
Development of a WAMS based test platform for power system real time transient stability detection and control
}

\author{
Jinquan Zhao ${ }^{*}$, Yujie Zhang ${ }^{1}$, Pan Zhang ${ }^{1}$, Xiaoming Jin $^{2}$ and Chao Fu
}

\begin{abstract}
The real-time transient stability detection and emergency control technology based on wide area response has become a hot research area in power system stability studies. Several different technologies have been proposed, but lots of problems remain to be solved before they can be applied in practice. A wide area measurement system (WAMS) based test platform is developed for transient stability detection and control. The design as well as main function modules of the platform are introduced. In addition, three generator power angle prediction methods and six response based transient instability detection technologies are given. Results of engineering application demonstrate that the developed test platform can provide a real-time operation environment, which can effectively compare and analyze the validity and practicability of these transient stability detection technologies. Based on the measured perturbed trajectories from actual power systems or the Real-Time Digital Simulators (RTDS), the platform can realize the assessment and visual result presentation of various responses from different transient instability detection technologies. The test platform can be applied to different power systems and it is convenient to embed new transient instability detection modules. Meanwhile some deficiencies and shortcomings in engineering application are pointed out and corresponding suggestions are given. In conclusion, the hardware and software structure, function modulus and engineering applications are presented. The application in actual power systems shows that it has a good application perspective.
\end{abstract}

Keywords: Test platform, Transient instability detection and control, PMU, WAMS

\section{Introduction}

With the wide application of phasor measurement units (PMU) and wide area measurement system (WAMS) in power system, the WAMS based power system real-time transient instability detection and emergency control has become a hot research area.

The real-time measurements based transient instability detection has been limited to the post-fault power angle trajectories for quite a long term. In reference [1-4], the power angle difference between any two generators is used as a transient instability indicator. If it exceeds a predefined threshold value, the power system is considered to enter transient instability status.

At present, there have been a lot of theoretical achievements in the field of the wide area response

\footnotetext{
* Correspondence: jqzhao2@tom.com

${ }^{1}$ College of Energy \& Electrical Engineering, Hohai University, Nanjing, China Full list of author information is available at the end of the article
}

based power system security and stability control technology [5-10]. Various generator power angle prediction techniques [11-14] and different transient instability detection technologies [15-18] were proposed. Reference [15] proposed an emergency EEAC based transient instability detection technique by using the measured trajectory. Reference [16] proposed a transient instability detection criterion based on the geometric feature of phase plane trajectory. Reference [17] proposed a transient instability detection technology by means of the transient unbalance energy variation rate of the most seriously perturbed generator. Reference [18] proposed a transient instability detection criterion based on perturbed voltage trajectory. However, the response-based transient instability detection technologies have not been applied in actual power systems, lots of problems need to be solved such as the integrity of wide area measurement information, the tolerance of bad data, the 
influence of data transmission delay, the accuracy and rapidity of detection as well as the emergency control schemes. Therefore it is necessary to analyze and compare the applicability and validity in actual power systems with different operating situations.

Considering that there has been no such test platform which can be used to compare and analyze different transient instability detection and control technologies in the real-time operating environment, a WAMS based power system transient stability detection and control test platform is developed. Several existing transient instability detection and control techniques are implemented on this platform. The platform realizes the comprehensive analysis and comparison of various detection techniques. and provides a test and simulation platform for these transient stability and control technologies based on WAMS before they are applied in actual power systems. The frame structure of the platform, main function modules and its engineering application are presented in this paper. Meanwhile some deficiencies and shortcomings in engineering application are pointed out and corresponding suggestions are given.

In Design of the platform, the design of the test platform is introduced. Observation substations selection module describes the implementation of main function modules including generator angle prediction, transient instability detection based on wide area response and emergency control. Engineering applications are presented in Data acquisition module. Observed substation access and monitoring module gives the conclusions.

\section{Design of the platform}

\section{Overall frame structure}

The WAMS based power system transient stability detection and control test platform mainly implements various response based transient instability detection techniques. The measured data can be obtained from the real-time data environment, such as actual power systems or real-time digital simulation platform (like RTDS).

The master station of our platform consists of basic modules and application modules. Basic modules include the front data collection module of PMU, the real-time sequential database, the historical sequential database and the relational database. The frame structure of WAMS modules is shown in Fig. 1. The temporal sequential database utilizes dedicated data storage model to provide better service of data storage and query.

The response based transient stability detection and control modules are integrated in the master station of our platform as part of the advanced application modules. In addition, application modules also include basic data modules such as real-time message of PMU and access to substations.

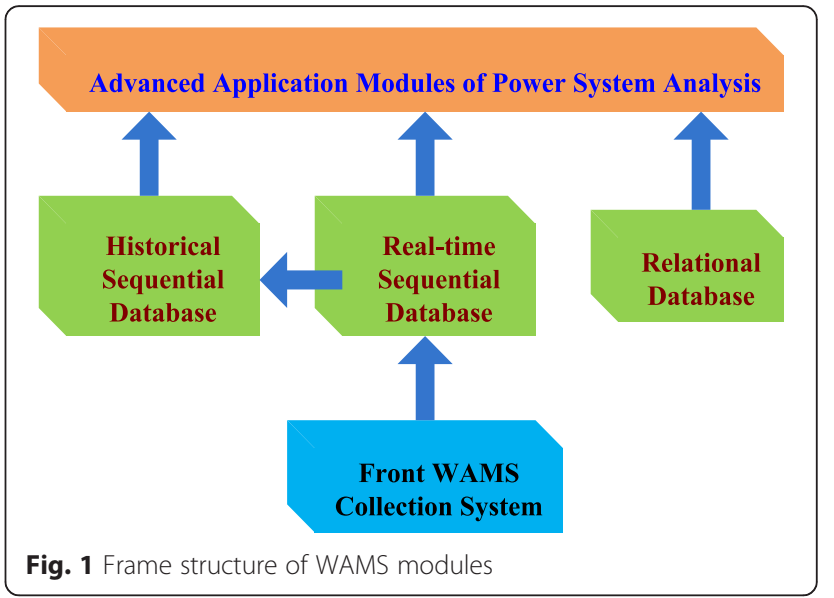

The main characteristics of the platform are summarized as follows.

- Implementing closed-loop test to evaluate different wide area response based power system transient instability detection and control technologies based on real-time data and offline contingency simulation.

- Providing a concise and intuitive visual interface to present real-time substation monitoring, generator power angle prediction curves, various transient instability detection results and platform parameters setting.

- Taking the most advantage of the service resources provided by WAMS including parameters and topological structure of the power system as well as real-time measured or historical storage data.

- Good data compatibility. It provides general data interface, supporting real-time measured data from actual power systems or RTDS platform and off-line results from simulation or other software.

- Good flexibility and extendibility. It provides open application programming interface (API) for power system operators or tester. It is convenient to update the existing modules or develop and add new ones to the platform.

\section{Hardware and software structure}

The test platform consists of hardware devices and software modules. The hardware and software structure diagram is shown in Fig. 2. Hardware includes the master server, the visual workstation and communication interface as well as substation actuating devices. Software modules include the data acquisition module, the station monitoring module, the generator power angle prediction module, the wide area response based transient 


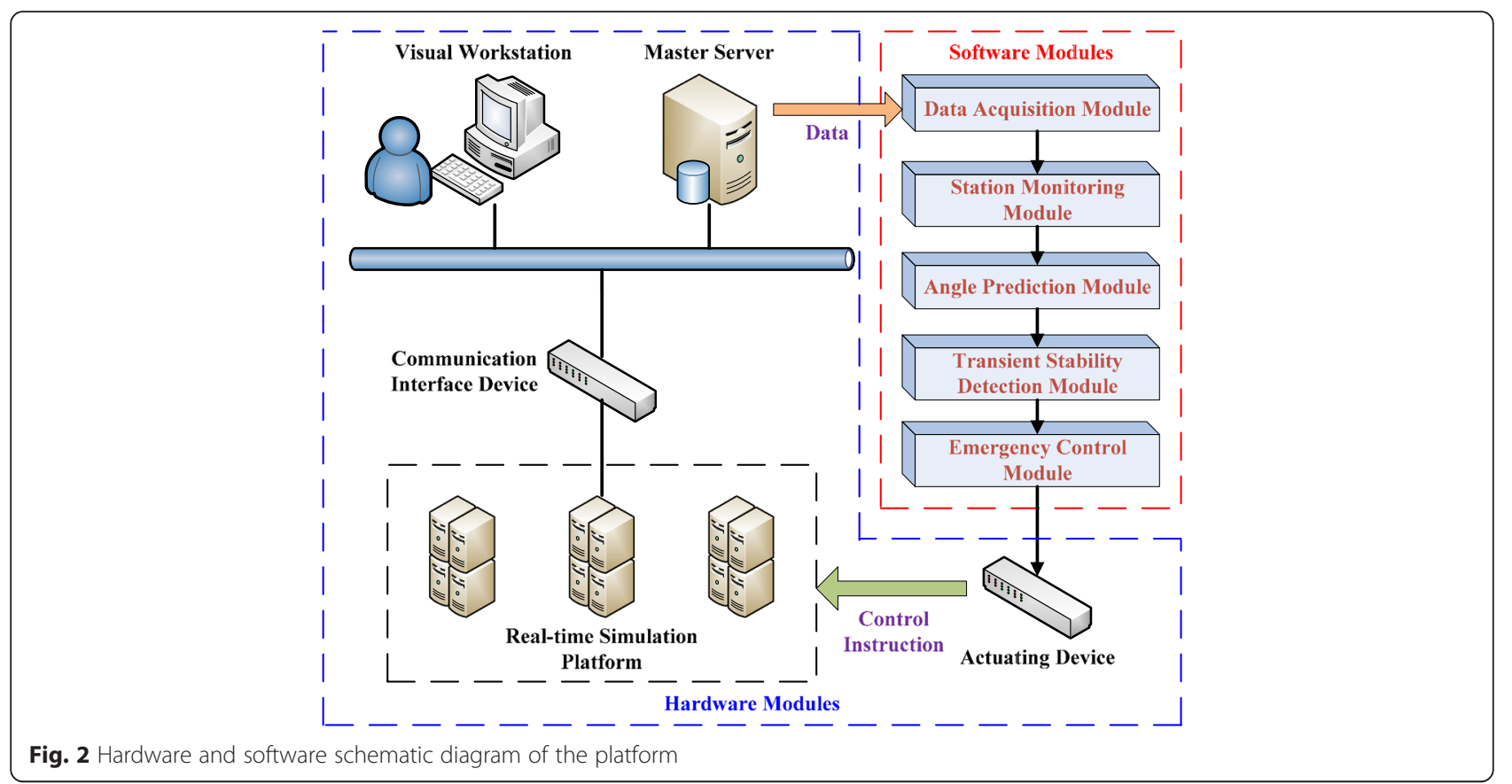

instability detection module and the emergency control module.

Software modules are integrated in the master station server to get data from the temporal sequential database. The workstation provides human-computer interactive operation and presentation of the test results. Through the communication interface, the real-time data is transmitted to the real-time sequential database located in the master server. The actuating device carries out the control instructions from the master server.

\section{Data interaction of the platform}

The advanced application modules of the platform consist of the online transient stability detection \& control subsystem offline history query \& contingency simulation subsystem. The data interaction diagram is shown in Fig. 3.

The general data interface module is developed to meet requirements of the online test and offline simulation. The interface supports real-time online data and offline data files exported from the RTDS platform or other time-domain simulation software. The real-time measured data and offline simulation data are stored in the real-time sequential database and then are transferred to the historical database. The former is measured directly by the front WAMS data collection device (PMU) and the latter is converted by the front WAMS data simulation module.

\section{Data and application programming interface}

Modules of the test platform are designed according to the principle with function decoupling and modularization. It provides an open and flexible development environment (namely 'plug and play') for power system designers and operators. In addition, A large amount of available data such as parameters and model of power systems (stored in relational database), graphics library for interface development and measured data acquisition interface can be utilized directly. Considering that the generator power angle prediction and response based transient instability detection modules share the same data and application programming interface, it is convenient to update the existing modules or develop new modules and add them to the test platform in order to meet the multiple test requirements.

\section{Main function modules}

1. Observation substations selection module The real-time measured data of power systems is tremendous. However, transient instability detection should be carried out with measurement data from the observed substations in actual engineering application, as less as possible. Thus, to determine which observed station is the most sensitive one to the disturbance characteristics is one of the most challenging problems concerned by the system operators. Therefore, the generator and bus substation selection module is designed, by which different generators and buses can be selected to formulate the observation scheme. Power system operators can analyze the applicability and validity of these transient instability detection technologies in different observation schemes. 


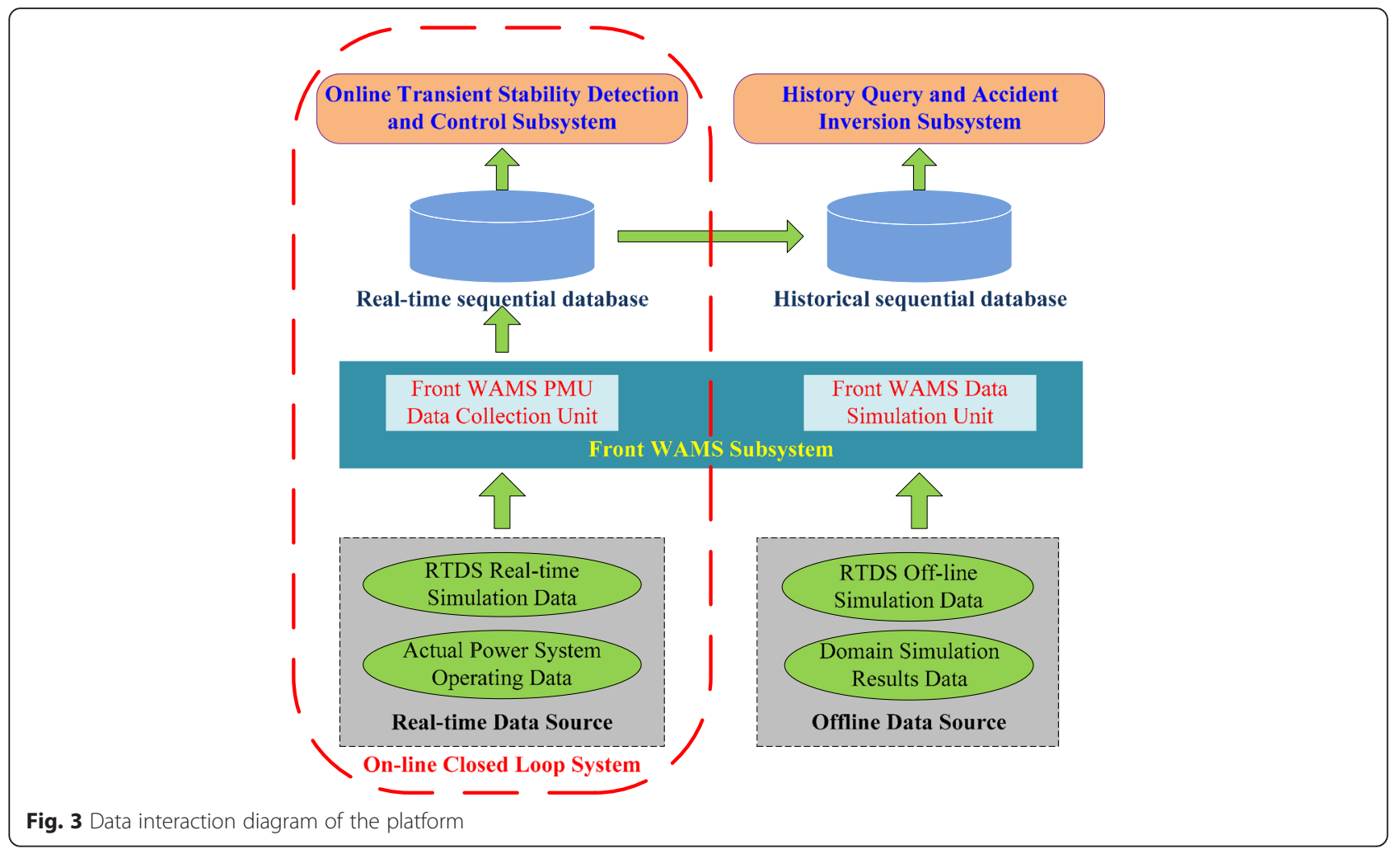

2. Data acquisition module

The data acquisition module of the test platform is designed to provide real-time measured data and historical simulation data for the advanced application modules. In addition, parameter and model as well as the topological structure information of the power system are also available. The list of the data provided by the platform is shown in Table 1.

3. Observed substation access and monitoring module To enable a wide application in different power systems, the substation access module is designed, by which new generators and bus substations can be accessed to the front WAMS collection devices. In

Table 1 List of the data provided by the platform

\begin{tabular}{|c|c|c|}
\hline Data & Attribution & Source \\
\hline Inertia constant & generator & relational database \\
\hline Base voltage & generator/bus & relational database \\
\hline Power angle & generator & $\begin{array}{l}\text { temporal sequential } \\
\text { database }\end{array}$ \\
\hline Mechanical power & generator & $\begin{array}{l}\text { temporal sequential } \\
\text { database }\end{array}$ \\
\hline Electric power & generator & $\begin{array}{l}\text { temporal sequential } \\
\text { database }\end{array}$ \\
\hline Three-phase voltage amplitude & bus & $\begin{array}{l}\text { temporal sequential } \\
\text { database }\end{array}$ \\
\hline
\end{tabular}

terms of different power systems, operators need to re-access the generators and bus substations and update the power grid parameters and model.

The observed substation monitoring module is designed to present the real-time curve and data monitoring of these observed generators and bus substations. The monitoring data are shown in Table 2.

\section{Methods}

Generator power angle prediction module

In order to analyze and study the influence of generator power angle prediction on transient instability detection, the power angle prediction module is designed with polynomial model based prediction, auto-regressive prediction method and trigonometric function based prediction [11-13].

Table 2 Monitoring data of generators and bus Stations

\begin{tabular}{ll}
\hline Station type & Monitoring data \\
\hline Observed generator & power angle \\
& mechanical power \\
& electric power \\
Observed bus & A-phase voltage amplitude \\
& B-phase voltage amplitude \\
& C-phase voltage amplitude
\end{tabular}


1. Polynomial model based prediction method The polynomial model for rotor angle prediction can be expressed as follows:

$\widehat{\delta}(t)=a_{0}+a_{1} t+a_{2} t^{2}+\cdots+a_{n} t^{n}$

where $\delta(t)$ is the predicted rotor angle at moment t. $A_{N}=\left[a_{0}, a_{1}, a_{2}, \cdots, a_{n}\right]^{T}$ is the parameter vector of the polynomial model. $n$ is the model order. Assuming $\Delta t$ as the sampling period, observation vector is $Y(N)=[\delta(0), \delta(\Delta t), \cdots \delta(N \Delta t)]^{T}$. The parameter vector can be solved by using the least square method as follows.

$A_{N}=P_{N} H^{T}(N) \cdot Y(N)$

Where

$H(N)=\left[\begin{array}{ccccc}1 & 0 & 0 & \cdots & 0 \\ 1 & \Delta t & (\Delta t)^{2} & \cdots & (\Delta t)^{n} \\ \cdots & \cdots & \cdots & \cdots & \cdots \\ 1 & N \Delta t & (N \Delta t)^{2} & \cdots & (N \Delta t)^{n}\end{array}\right]$

$P_{N}=\left[H^{T}(N) H(N)\right]^{-1}$

If vector $A_{N}$ is obtained, the value of rotor angle in the future can be calculated by Eq. (5).

$$
\begin{aligned}
\widehat{\delta}(k \Delta t) & =a_{0}+a_{1} k \Delta t+\cdots+a_{n}(k \Delta t)^{n} \\
k & =N+1, N+2, \cdots, N+l
\end{aligned}
$$

where $l$ is the number of predicted data.

When the new rotor angle measurement is updated, the rolling prediction method can be used to form the new observation vector $Y(N+1)$ and calculate the new parameter vector $A_{N+1}$ by Eq. (2). Matrix $H(N)$ maintains unchanged since it is irrelevant with the measurements.

2. Trigonometric function model based prediction method

The trigonometric function model for the perturbed rotor angle trajectory prediction can be described as.

$\delta(t)=\sum_{n=0}^{\infty} a_{n} \cos n t+b_{n} \sin n t$

where $n$ is the model order, $A_{N}=\left[a_{0}, a_{1}, b_{1}, \cdots, a_{n}, b_{n}\right]^{T}$ is the parameter vector of the trigonometric function model.

The measurement matrix can be expressed as.

$$
H(N)=\left[\begin{array}{cccccc}
1 & 1 & 0 & 1 & 0 & \cdots \\
1 & \cos (\Delta t) & \sin (\Delta t) & \cos (2 \Delta t) & \sin (2 \Delta t) & \cdots \\
1 & \cos (2 \Delta t) & \sin (2 \Delta t) & \cos (4 \Delta t) & \sin (4 \Delta t) & \cdots \\
\cdots & \cdots & \cdots & \cdots & \cdots & \cdots \\
1 & \cos (N \Delta t) & \sin (N \Delta t) & \cos (2 N \Delta t) & \sin (2 N \Delta t) & \cdots
\end{array}\right]
$$

If parameter vector $A_{N}$ is estimated by using the least square method as Eq. (2), the predicted values of rotor angle are calculated by Eq. (8).

$$
\begin{aligned}
\delta(k \Delta t) & =\sum_{n=0}^{\infty} a_{n} \cos n(k \Delta t)+b_{n} \sin n(k \Delta t) \\
k & =N+1, N+2, \cdots, N+l
\end{aligned}
$$

where $l$ is the number of predicted data.

As same as the polynomial model based prediction method, if a new measurement is obtained, the measurement vector is updated and then the latest predicted value can be figured out by rolling prediction.

3. Auto regression model based prediction method The auto regression model of the generator angle can be written as.

$$
\widehat{\delta}(t)=\alpha_{1} \delta(t-1)+\alpha_{2} \delta(t-2)+\cdots+\alpha_{n} \delta(t-n)
$$

where

$A_{N}=\left[a_{1}, a_{2}, \cdots, a_{n}\right]^{T}$ is the parameter vector.

$\phi_{t}=[\delta(t-1), \delta(t-2), \cdots, \delta(t-n)]^{T}$ is the measured

rotor angle vector, $n$ is the model order.

The observation vector can be written as $Y(N)=$ $[\delta(1), \delta(2), \cdots, \delta(N)]^{T}$.

Parameter vector $A_{N}$ could be calculated by Eq. (2). The observation matrix is.

$H(N)=\left[\begin{array}{lllc}\delta(0) & \delta(-1) & \cdots & \delta(1-n) \\ \delta(1) & \delta(0) & \cdots & \delta(2-n) \\ \cdots & \cdots & \cdots & \cdots \\ \delta(N-1) & \delta(N-2) & \cdots & \delta(N-n)\end{array}\right]$

Finally, the predicted value of generator angle can be obtained by Eq. (9).

$$
\begin{aligned}
& \widehat{\delta}(N+1)=a_{1} \delta(N)+a_{1} \delta(N-1)+\cdots+a_{n} \delta(N+1-n) \\
& \delta(N+2)=a_{1} \delta(N+1)+a_{1} \delta(N)+\cdots+a_{n} \delta(N+2-n) \\
& \cdots \\
& \hat{\delta}(N+l)=a_{1} \delta(N+l-1)+a_{1} \delta(N+l-2)+\cdots \\
& +a_{n} \delta(N+l-n)
\end{aligned}
$$

where $l$ is the number of predicted data.

Once the latest data $\delta(N+1)$ is obtained, the rolling least square method can be used to update relevant parameter as follows.

$$
\begin{aligned}
K_{N+1} & =\frac{P_{N} \phi_{N+1}}{1+\phi_{N+1}{ }^{T} P_{N} \phi_{N+1}} \\
A_{N+1} & =A_{N}+K_{N+1}\left[\delta(N+1)-\phi_{N+1}^{T} A_{N}\right] \\
P_{N+1} & =P_{N}-K_{N+1 \phi N+1}{ }^{T} P_{N}
\end{aligned}
$$




\section{Transient instability detection module}

In this section, the implementation of seven transient instability detection technologies will be briefly described. Input data and threshold values of these transient instability detection technologies are shown in Table 3.

1. Maximum power angle difference based detection module

The power angle difference is usually taken as a criterion for transient instability detection in traditional engineering application. In this module, the generator power angle is predicted by one of

Table 3 List of the input of all Criterions

\begin{tabular}{|c|c|c|}
\hline Criterion name & Input data & Source \\
\hline \multirow{3}{*}{$\begin{array}{l}\text { Maximum power angle } \\
\text { difference based criterion }\end{array}$} & observed generators & system operator \\
\hline & angle difference & system operator \\
\hline & power angle & WAMS \\
\hline \multirow{6}{*}{$\begin{array}{l}\text { Clearance angle of } \\
\text { homology generators } \\
\text { group based criterion }\end{array}$} & observed generators & system operator \\
\hline & clearance angle difference & system operator \\
\hline & power angle & WAMS \\
\hline & mechanical power & WAMS \\
\hline & electric power & WAMS \\
\hline & inertia constant & WAMS \\
\hline \multirow{6}{*}{$\begin{array}{l}\text { Convexity of trajectory in } \\
\text { the phase plane based } \\
\text { criterion }\end{array}$} & observed generators & system operator \\
\hline & disturbance information & WAMS \\
\hline & power angle & WAMS \\
\hline & mechanical power & WAMS \\
\hline & electric power & WAMS \\
\hline & inertia constant & WAMS \\
\hline \multirow{6}{*}{$\begin{array}{l}\text { Measured trajectory } \\
\text { based EEAC criterion }\end{array}$} & observed generators & system operator \\
\hline & disturbance information & WAMS \\
\hline & power angle & WAMS \\
\hline & mechanical power & WAMS \\
\hline & electric power & WAMS \\
\hline & inertia constant & WAMS \\
\hline \multirow{6}{*}{$\begin{array}{l}\text { Single generator energy } \\
\text { function based criterion }\end{array}$} & observed generators & system operator \\
\hline & disturbance information & WAMS \\
\hline & power angle & WAMS \\
\hline & mechanical power & WAMS \\
\hline & electric power & WAMS \\
\hline & inertia constant & WAMS \\
\hline \multirow{6}{*}{$\begin{array}{l}\text { Perturbed voltage } \\
\text { trajectory } \omega_{k} \text { integration } \\
\text { based criterion }\end{array}$} & observed bus stations & system operator \\
\hline & $\begin{array}{l}\text { threshold value integration } \\
\text { starting voltage }\end{array}$ & system operator \\
\hline & $\begin{array}{l}\text { threshold value instability } \\
\text { detection integration }\end{array}$ & system operator \\
\hline & phase-A voltage amplitude & WAMS \\
\hline & phase-B voltage amplitude & WAMS \\
\hline & phase-B voltage amplitude & WAMS \\
\hline
\end{tabular}

the angle prediction methods mentioned above according to the perturbed power angle of the observed generators. If the predicted power angle difference between any two generators exceeds a predefined threshold value, then the power system is considered as transient instable.

2. Clearance angle of homology generators group based detection module

Power system transient instability usually can be manifested as two generator cluster instability mode. In this module, the observed generators are divided into two generator groups based on power angle. The equivalent parameters of the two generator groups can be computed by.

$\delta_{A}=\frac{\sum_{i \in A}^{n_{A}} M_{i} \delta_{i}}{\sum_{i \in A}^{n_{A}} M_{i}}, \delta_{B}=\frac{\sum_{j \in B}^{n_{B}} M_{j} \delta_{j}}{\sum_{j \in B}^{n_{B}} M_{j}}$

$\left|\delta_{A}-\delta_{B}\right|>\delta_{\text {set }}$

where $\delta_{A}$ and $\delta_{B}$ are respectively the equivalent power angle of the leading generator group $A$ and the lagging generator group $B, \delta_{\text {set }}$ is the threshold value of transient instability detection, $M_{i}$ and $M_{j}$ are the inertia constant of generator $i$ and $j$. If Eq. (16) is established, power system can be considered as transient instable.

3. Measured trajectory based EEAC detection module Based on the EEAC methodology, an emergency EEAC method to predict, analyze and estimate the degree of transient stability was proposed in

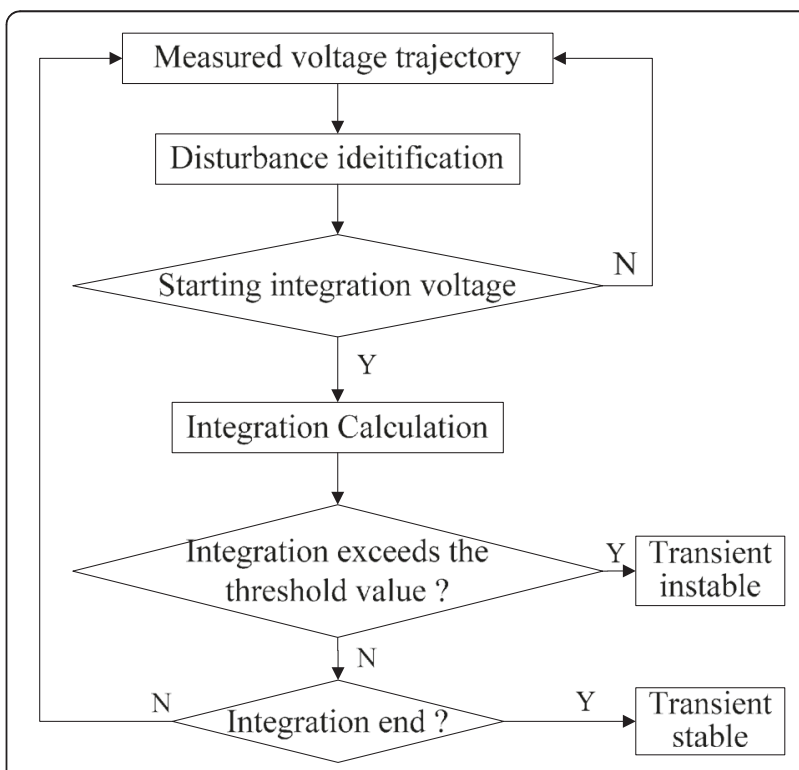

Fig. 4 Flow chart of voltage trajectory based detection criterion 
reference [15]. In this module, the observed generators are divided into two generator groups. Furthermore, the two generator groups are equivalent to a single machine infinite bus (SMIB) system. The transient stability is detected by searching the dynamic saddle point (DSP) and the farthest point (FEP) of the power angle-unbalanced power curve of SMIB. If the current operating status reaches the DSP, the system can be considered as transient instable. Otherwise the system can be considered as transient instable if FEP is reached.

4. Convexity of trajectory in the phase plane based detection module A transient instability detection method based on the trajectory geometry characteristics in phase plane was proposed in reference [16]. Based on the OMIB system, three indexes are calculated by Eqs. $(17,18,19,20$ and 21$)$ including the convexity index in the phase of angle-angle acceleration $\left(K_{1}\right)$, the convexity index in the phase of unbalanced powerangle $\left(K_{2}\right)$ and the changing rate of the unbalanced electric power $\left(K_{3}\right)$. When these three indexes are positive at the same time, the system is considered as transient instable.

$k_{i}(t)=\frac{\omega_{i}(t)-\omega_{i}(t-1)}{\delta_{i}(t)-\delta_{i}(t-1)}$

$K_{1}=\Delta k_{i}(t)=k_{i}(t)-k_{i}(t-1)$

$\frac{d \Delta P(\delta)}{d \delta}=\frac{\Delta P(t)-\Delta P(t-1)}{\delta(t)-\delta(t-1)}$

$K_{2}=\frac{d^{2} \Delta P(\delta)}{d \delta^{2}}=\frac{\Delta P^{\boxplus}(t)-\Delta P^{\bigotimes}(t-1)}{\delta(t)-\delta(t-1)}$
$K_{3}=\frac{d \Delta P(t)}{d t}=\frac{\Delta P(t+1)-\Delta P(t)}{\Delta t}$

where $\Delta \delta_{i}(t), \Delta \omega_{i}(t)$ and $\Delta P(t)$ are measured values of generator power, angle angular velocity and unbalanced electric power at moment $t$, respectively.

5. Single generator energy function based detection module

A transient energy changing rate based transient instability detection method was proposed in reference [17]. It identifies the most severely perturbed generator based on relative kinetic energy of generators and electric power reflecting the change of power system. According to energy changing rate and power angle acceleration of the most severely perturbed generator, transient stability of power system can be detected. The variation rate of transient energy can be computed by Eq. (22).

$\frac{d V_{i}}{d t}=H_{i} \bar{\omega}_{i} \frac{d \bar{\omega}_{i}}{d t}-\left(P_{m i}-P_{e i}-\frac{H_{i}}{H_{T}} P_{C O I}\right) \bar{\omega}_{i}$

where $V_{i}$ is the energy function of generator $i, P_{C O I}$ is the unbalanced power of the system center of inertia (COI), $H_{i}$ is the inertia of generator $i, \bar{\omega}_{i}$ is the angular velocity of generator $i, H_{T}$ is the sum inertia of all generators, $P_{m i}$ and $P_{e i}$ are mechanical power and electromagnetic power of generator $i$, respectively.

6. Perturbed voltage trajectory integration criterion based detection module

A technique for predicting transient stability based on perturbed voltage trajectories was presented in reference [18]. The main steps are explained as follows and the detailed algorithm flow chart is shown in Fig. 4.

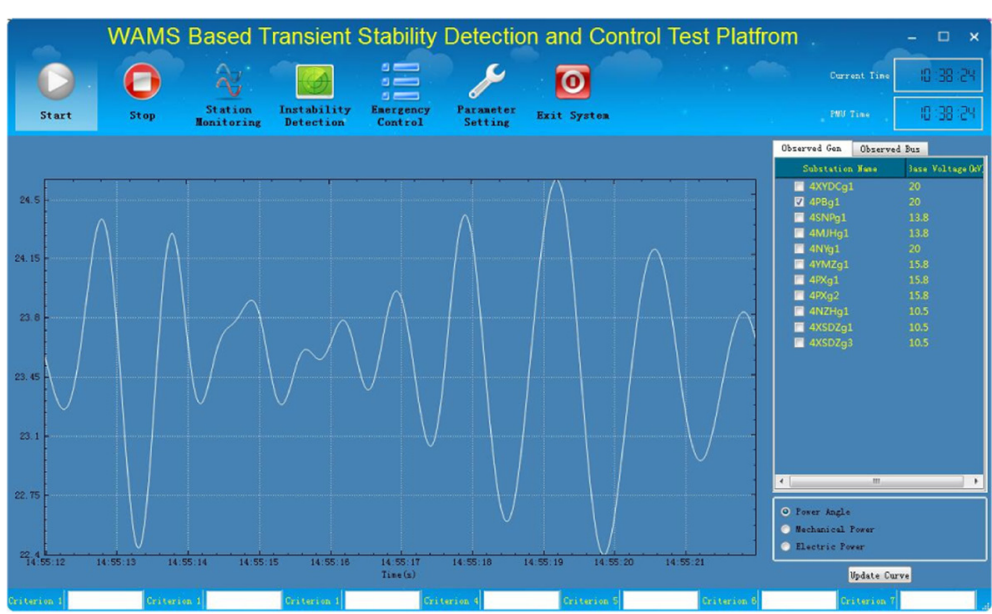

Fig. 5 Main interface of the test platform 
Table 4 Assessment information of all methods

\begin{tabular}{|c|c|c|c|}
\hline Criterion name & Instability detection moment (s) & Parameters & Values \\
\hline \multirow{6}{*}{$\begin{array}{l}\text { Maximum power angle difference } \\
\text { based criterion }\end{array}$} & \multirow[t]{6}{*}{1.54} & threshold value of power angle difference () & 180 \\
\hline & & power angle difference detecting instability () & 180.58 \\
\hline & & leading generator & Yemazhai\# 1 \\
\hline & & lagging generator & Nayong\#1 \\
\hline & & maximum power angle difference $\left(^{\circ}\right)$ & 357.91 \\
\hline & & maximum power angle difference moment (s) & 2.72 \\
\hline \multirow{2}{*}{$\begin{array}{l}\text { Clearance of generator cluster based } \\
\text { criterion }\end{array}$} & \multirow[t]{2}{*}{2.02} & threshold value of clearance angle difference $\left(^{\circ}\right)$ & 150 \\
\hline & & clearance of generator cluster detecting instability $\left(^{\circ}\right)$ & 156.7528 \\
\hline \multirow[t]{2}{*}{ Measured trajectory based EEAC } & \multirow[t]{2}{*}{1.72} & unbalanced power (MW) & 15.8657 \\
\hline & & power angle speed (rad/s) & 3.0980 \\
\hline \multirow{3}{*}{$\begin{array}{l}\text { Trajectory convexity in the phase } \\
\text { plane based criterion }\end{array}$} & \multirow[t]{3}{*}{2.28} & convexity index in the angle-angle acceleration phase & 0.0005 \\
\hline & & convexity index in the unbalanced power-angle phase & 0.3162 \\
\hline & & changing rate of electric power & 24.3630 \\
\hline \multirow{2}{*}{$\begin{array}{l}\text { Single generator energy function } \\
\text { based criterion }\end{array}$} & \multirow[t]{2}{*}{1.96} & power angle acceleration $\left(\mathrm{rad} / \mathrm{s}^{2}\right)$ & -0.0041 \\
\hline & & energy changing rate (MW/s) & 282.7081 \\
\hline \multirow{4}{*}{$\begin{array}{l}\text { Perturbed voltage trajectory integration } \\
\text { based criterion }\end{array}$} & \multirow[t]{4}{*}{0.74} & key bus station & Shuanglong \\
\hline & & voltage upper limit (pu) & 0.85 \\
\hline & & threshold value of voltage integration & 0.03 \\
\hline & & voltage integration value & 0.0304 \\
\hline
\end{tabular}

Step1 Obtaining the voltage measurements (trajectories) by PMU/WAMS and detecting the disturbance (fault occurs and fault clears).

Step2 Determining the voltage trajectory used for integration and calculating the integration based on the perturbed voltage trajectory by Eq. (23).

$$
\left\{\begin{array}{l}
A_{1}=\int_{t_{0}}^{t_{\text {end }}}\left[V_{U}-V(t)\right]\left|\frac{V(t-T)-V(t)}{T}\right| d t \\
V(t)<V_{U}
\end{array}\right.
$$

Step3 When the integration value exceeds the given threshold value at a certain time, the power system is unstable. If the voltage trajectory returns above the voltage upper limit and maintains a phase of time, the system is detected as transient stable.

\section{Closed-loop emergency control module}

In this module, the closed-loop emergency control module is designed. The sort list (descending order) of the observed generators according to power angle is given, by which operators can select generators to formulate the emergency control schemes including the control site and quantity. Different emergency control schemes in different operation conditions and failure situations can be tested and verified through the platform.

\section{Results}

The WAMS based power system transient stability detection and control test platform is developed by QT. It has been applied in China Southern Power Grid Research

Table 5 Platform Test Results of The Fault Cases of China Southern Power Grid

\begin{tabular}{|c|c|c|c|c|}
\hline \multirow[t]{2}{*}{ Criterion name } & \multicolumn{4}{|c|}{ Transient stability detection moment (s) } \\
\hline & Case 1 & Case 2 & Case 3 & Case 4 \\
\hline Maximum power angle difference based criterion & 1.86 & 1.22 & 1.96 & 0.68 \\
\hline Clearance of generator cluster based criterion & 2.6 & 1.58 & 1.04 & 0.66 \\
\hline Measured trajectory based EEAC & 1.40 & 1.16 & 1.40 & 0.88 \\
\hline Trajectory convexity in the phase plane based criterion & 1.18 & 1.06 & 1.00 & 0.84 \\
\hline Single generator energy function based criterion & 0 & 2.76 & 3.58 & 1.58 \\
\hline Perturbed voltage trajectory integration based criterion & 0.96 & 1.10 & 0.80 & 0.56 \\
\hline
\end{tabular}


Table 6 Detection information of the observed substations in the tie-line of Guangdong and Guangxi

\begin{tabular}{lcccc}
\hline $\begin{array}{l}\text { Observed } \\
\text { stations }\end{array}$ & \multicolumn{4}{l}{ Transient instability detection moment (s) } \\
\cline { 2 - 5 } & Case 1 & Case 2 & Case 3 & Case 4 \\
\hline Liudong & 1.38 & 1.28 & 1.52 & 0.56 \\
Guilin & 0.96 & 1.10 & 0.80 & 1.16 \\
Hezhou & 1.28 & 1.32 & 1.56 & 0.60 \\
Wuzhou & 1.12 & 1.26 & 1.28 & 2.26 \\
Xianlingshan & 1.20 & 1.20 & 1.32 & 0.70 \\
\hline
\end{tabular}

Institute and played an important role in some engineering applications. In this section, parts of the analysis and comparison results are presented. The main interface of the platform is shown in Fig. 5.

\section{Determination of the system protection scheme in Liupanshui power grid}

The test platform developed in this paper has played an important role in the formulation of the system protection schemes of Liupanshui power grid (in Guizhou province).

Based on the operation data in the year 2014 of China Southern Power Grid provided by RTDS platform (the measured cycle is $0.02 \mathrm{~s}$ ), 14 typical fault (102 cases in total) in Liupanshui power grid are selected to analyze the validity and applicability of the response based transient instability detection technologies mentioned above. Studies cover various conditions including different observation scheme of generators and substations (22 observable generators and 28 observable substations), different threshold values, different hybrid power generation with fire-water-wind, the asymmetric and nonmetallic three-phase short circuit faults. One of the test results is given in Table 4.

By means of testing and analyzing all cases through our test platform, the power system operators can make a comprehensive comparison of the accuracy, rapidity and the project implementation of all these response based transient instability detection technologies. It is determined that the 'Perturbed Voltage Trajectory Integration Based Criterion' is adopted to the project demonstration of the Liupanshui power grid and four $220 \mathrm{kV}$ substations are taken as the observed substations of the special protection system including Shuanglong, Huangjiashan, Taisha and Liuzhi.

\section{Case analysis of the Guangdong-Guangxi Tie-lines}

In order to illustrate the validity and practicality of the test platform, 50 fault cases of the tie-lines between Guangdong and Guangxi are analyzed. Part of the test results are shown in Table 5.

Research shows that generators of Guangdong and Guangxi will lose synchronization when there happens to be DC-blocking faults or short-circuit faults. The oscillation center is always located in the tie-line of Guangdong and Guangxi. Detection results of the observed $500 \mathrm{kV}$ bus stations are shown in Table 6.

\section{Discussion}

Currently the PMU data are generally transmitted by the dispatching data communication network based on TCP/IP. The average delay of data transmission is close to 100 milliseconds and is uncertain. Meanwhile, the data buffer period of the WAMS master station system is generally more than $1 \mathrm{~s}$ and even close to $2 \mathrm{~s}$. In addition, the time consuming of response based transient instability detection calculation is evitable. The detailed delay distribution is described as shown in Fig. 6. Thus, the existing PMU/WAMS is mainly used for the real-time online monitoring of the power system dynamic process. The reliability and rapidity cannot meet the requirement of the fast and real-time wide area control.

At present, power system stability control system is still based on the embedded stability control devices,

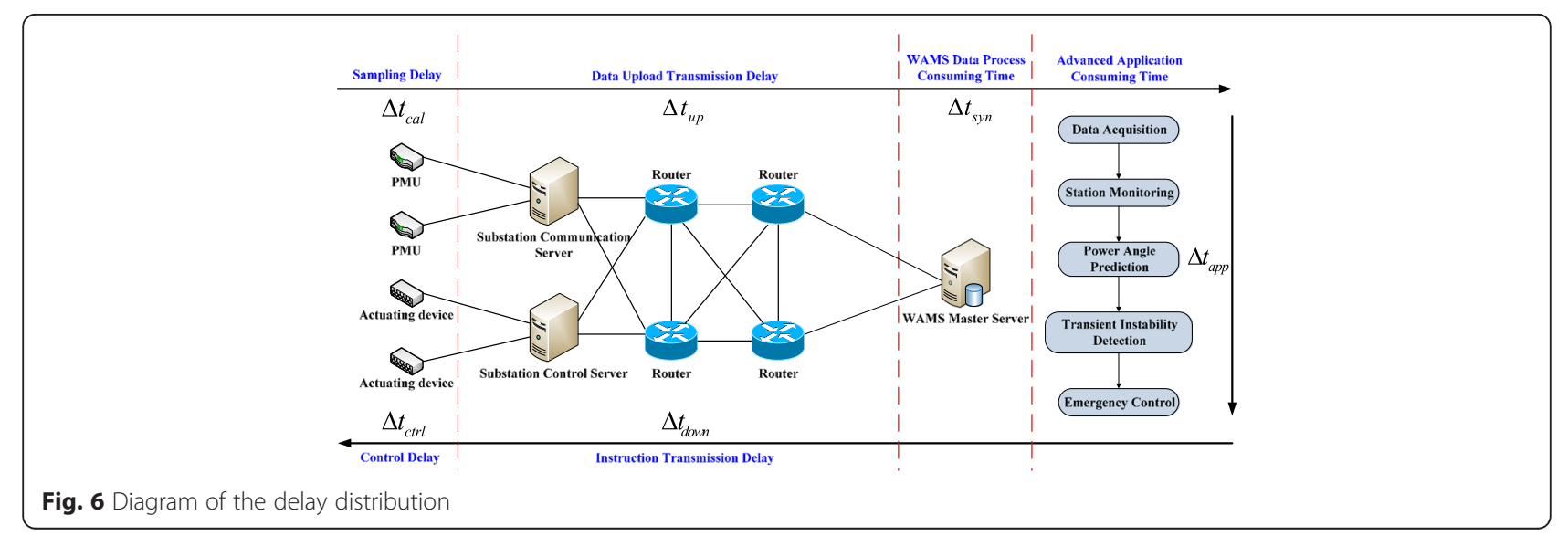


which has several fixed observation control substations. With the development of PMU/WAMS, transient instability and control system based on WAMS can be carried out based on various WAMS substations. The task of calculation in master station can be decomposed into several sub-tasks which can be allocated to various distributed WAMS substations.

\section{Conclusions}

Power system security and stability control technology based on wide area response is currently a research hotspot. According to existing research achievements, a WAMS based test platform is developed for power system transient instability detection and control. The platform implements various response based transient instability detection and control technologies in real-time data environment and provides visual presentation of results. Furthermore, the platform has good flexibility and extendibility. Data support, application integration and test environment are provided. The general data and application programming interface (API) is available by which testers or operators can add new transient instability detection modules to the platform.

Application in actual engineering demonstrates that this platform can meet the requirements of testing and analyzing in different operation conditions and failure situations. It is an effective test platform to analyze and compare the response based transient instability detection and control technologies. It can provide a reference for actual engineering application, which has a practical significance in terms of the application of the response based transient instability detection and control technologies in actual power systems.

\section{Acknowledgements \\ Project Supported by National Natural Science Foundation of China (51577049); Open Foundation of State Key Lab. of Alternate Electrical Power System with Renewable Energy Sources (Grant No. LAPS14005)".}

\footnotetext{
About the authors

Jinquan Zhao (M'2006) was born in Yangquan, Shanxi province, China, on June 26 1972. He received the B.S. and Ph.D. degrees, all in electrical engineering, from Shanghai Jiao Tong University, Shanghai, China, in 1993 and 2000, respectively. From December 2000 to September 2003, he was a post-doctoral associate in Cornell University, Ithaca, NY. He was a postdoctoral associate in Tsinghua University, Beijing, China. Currently he is a professor in College of Energy \& Electrical Engineering, Hohai University, Nanjing, China. His researches include voltage stability analysis and control, OPF and its applications.

Yujie Zhang received the B.S. in electrical engineering from Nanjing University of Technology, Nanjing, China, in 2013. Currently he is a M.S. student in College of Energy \& Electrical Engineering, Hohai University, Nanjing, China. His research interest is power system stability and control. Pan Zhang received the B.S. in electrical engineering from Hohai University, Nanjing, China, in 2013. Currently he is a M.S. student in College of Energy \& Electrical Engineering, Hohai University, Nanjing, China. His research interest is power system transient stability and control. Xiaoming Jin received the B.S. in electrical engineering from Xi'an Jiaotong University, Xi'an, China, in 1983. Currently he is a senior technical expert
}

in Southern Power Grid, Guangzhou, China. His researches include power system planning and Direct-Current (DC) transmission technology. Chao Fu received the B.S. and M.S. in electrical engineering from Xi'an Jiaotong University, Xi'an, China, in 2004 and 2007, respectively. Currently he is an engineer at China Southern Power Grid Science Research Institute and his researches include power system planning as well as power system stability and control.

\section{Authors' contributions}

ZJ carried out the wide area response based power system transient stability detection and control studies. ZY carried out the design and implementation of the test platform and drafted the manuscript. ZP participated in the development of test platform. JX and FC provided simulation data and suggestions for improvement of test platform. All authors read and approved the final manuscript.

\section{Competing interests}

The authors declare that they have no competing interests.

\section{Author details}

${ }^{1}$ College of Energy \& Electrical Engineering, Hohai University, Nanjing, China. ${ }^{2}$ Electric Power Research Institute, CSG, Guangzhou, China.

Received: 10 May 2016 Accepted: 10 May 2016

Published online: 20 June 2016

\section{References}

1. Takahashi, M., Matsuzawa, K., Sato, M., et al. (1988). Fast generation shedding equipment based on the observation of swings of generators. IEEE Transactions on Power Systems, 3(2), 439-446.

2. Liu, C. W., \& Thorp, J. (1995). Application of synchronized phasor measurements to real-time transient stability prediction. IEE Proc. Gener. Transm. Distrib., 142(4), 355-360.

3. Stanton, S. E., Slivinsky, C., Martin, K., et al. (1995). Application of phasor measurements and partial energy analysis in stabilizing large disturbances". IEEE trans. On power systems, 10(1), 297-306.

4. Liu, C. W., \& Thorp, J. S. (2000). New methods for computing power system dynamic response for real-time transient stability prediction. IEEE Trans. On Circuits and Systems: Fundamental Theory and application, 47(3), 324-337.

5. Daoud, A., Karady, G., Amin, A. (2001). "A new fast-learning algorithm for predicting power system stability," Proceedings of 2001 IEEE PES winter meeting, Jan. 28-Feb. 1, Singapore: 594-598.

6. Cai, G., Chan, K. W., Mu, G., et al. (2004). Transient instability detection using line transient potential energy. Electric Power Components \& Systems., 32(6), 627-643.

7. De La Ree, J., Centeno, V., Thorp, J., \& Phadke, A. G. (2010). Synchronized phasor measurement applications in power systems. IEEE Trans. on Smart Grid, 1(1), 20-27.

8. Rajapakse, A. D., Gomez, F. R., Nanayakkara, K., et al. (2010). Power angle instability prediction using post-disturbance voltage trajectory. IEEE Trans. on Power Systems, 25(2), 947-956.

9. Terzija, V., Valverde, G., Cai, D., et al. (2011). Wide-area monitoring, protection, and control of future electric power networks. Proceedings of the IEEE., 99(1), 80-93.

10. Taylor, C. W., Erickson, D. C., Martin, K. E., et al. (2005). WACS-Wide-Area Stability and Voltage Control System: R\&D and Online Demonstration. Proceedings of IEEE., 93(5), 892-906.

11. Mao, A., Guo, Z., \& Zhang, X. (2006). A fast transient stability predicting method based on the WAMS process measurement data. Proceedings of CSEE., 26(17), 38-43 (in Chinese).

12. Song, F., Bi, T., \& Yang, Q. (2006). Perturbed trajectory prediction method based on wide area measurement systems. Automation of Electric Power Systems., 30(23), 27-31 (in Chinese).

13. Li, G. (1994). Study on prediction control for transient stability of power systems. Automation of Electric Power Systems., 18(3), 25-31 (in Chinese).

14. Wu, X., Zhao, J., Xu, A., et al. (2011). Review on transient stability prediction methods based on real time wide-area phasor measurements (in Proc. IEEE- DRPT 4th Int. Conf., China, pp. 320-326).

15. Xue, Y. (1998). "Fast analysis of stability using EEAC and simulation technologies," in Proceedings of IEEE POWERCON 98. Beijing, China, August, pp. 12-16. 
16. Xie, H., Zhang, B. (2008). "Power system transient instability detection algorithm based on real time measurement," in IEEE Asia Pacific Conference on Circuits and Systems, Macao, China, November, pp. 631-634.

17. Song, F., Bi, T., and Yang, Q. (2006). "A Novel Transient stability Analysis Method for Multi-machine System," in The 7th IET International Conference on Advances in Power System Control, Hongkong, China, October, pp. 450-456.

18. Zhao, J., Li, J., Wu, X., et al. (2011). A novel real-time transient stability prediction method based on post-disturbance voltage trajectories (The International Conference on Advanced Power System Automation and Protection, Beijing, China, pp. 14-16).

\section{Submit your manuscript to a SpringerOpen ${ }^{\mathcal{O}}$ journal and benefit from:}

- Convenient online submission

- Rigorous peer review

- Immediate publication on acceptance

- Open access: articles freely available online

- High visibility within the field

- Retaining the copyright to your article

Submit your next manuscript at $\boldsymbol{\wedge}$ springeropen.com 